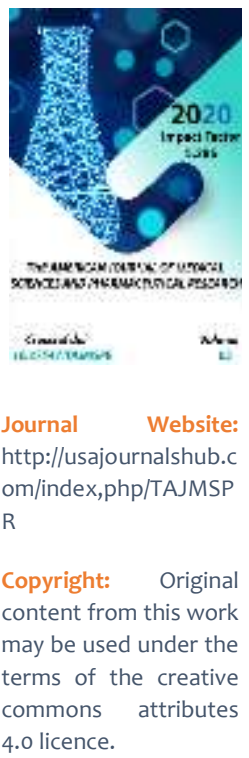

\section{Lupus Nephritis In Systemic Lupus Erythematosus}

\author{
Nazarova Nigina Otabek Qizi \\ Assistant, Department Of Faculty And Hospital Therapy No. 2, Tashkent Medical Academy, \\ Tashkent, Uzbekistan \\ Jabbarov Azim Atakhanovich \\ Associate Professor, MD, Department Of Faculty And Hospital Therapy No. 2 Tashkent \\ Medical Academy, Tashkent, Uzbekistan \\ Abduhalikova Nigora Fahriddinovna \\ Trainee Researcher, Normal And Pathological Physiology Tashkent Medical Academy, \\ Tashkent, Uzbekistan \\ Madazimova Dilrux Xayotjonovna \\ Assistant, Department Of Faculty And Hospital Therapy No. 2 Tashkent Medical Academy, \\ Tashkent, Uzbekistan
}

\title{
ABSTRACT
}

The pathogenesis, clinic, and treatment of kidney damage in patients with systemic lupus erythematosus (SLE) are considered. It is noted that if at the beginning of the disease signs of kidney damage are present in $25-50 \%$ of SLE patients, then later they are detected in almost $60 \%$ of adults and $80 \%$ of children. Variants of kidney damage in SLE are described.

The pathogenesis of SLE is generally considered on the model of lupus nephritis. The morphological classification of lupus nephritis, features of the main nephrological syndromes, and clinical variants (active and inactive) are presented. It is indicated that the treatment strategy depends on the activity of the disease, the clinical and morphological variant of lupus nephritis.

\section{KEYWORDS}

SLE, lupus nephritis, immune response

\section{INTRODUCTION}

Kidney damage in systemic lupus erythematosus (SLE) remains one of the most common, severe and prognostically important. The possibilities of modern immunosuppressive therapy, on the one hand, have reduced the proportion of patients with end-stage renal failure, and on the other hand, they have demonstrated the prognostic 
importance of kidney damage for the course of the disease as a whole [1-5]. In a random sample, $25-50 \%$ of patients with SLE have signs of kidney damage at the beginning of the disease, and later they are diagnosed in almost $60 \%$ of adults and $80 \%$ of children $[1,5]$. Kidney damage in SLE is currently multi-faceted (table 1).

Table

1. Variants of kidney damage in SLE

1. LUPUS NEPHRITIS

2. INTERSTITIAL NEPHRITIS

\section{VASCULAR LESIONS}

\section{THE MAIN RESULTS AND FINDINGS}

There is no doubt that the given classification is conditional. It is rare to find morphological and clinical signs of only one of the listed variants. SLE is characterized not only by multifaceted kidney damage, but also by the transformation of one variant into another during the course of the disease. This applies both to the morphological classes of lupus nephritis itself, and to the combination or independent development of nephropathy caused by vascular lesions [6-8]. Lupus nephritis is a paradigm (model) of immunocomplex inflammation, the mechanism of development of which reflects the pathogenesis of SLE in General. The basis of the disease is polyclonal hyperactivity of the B-cell system, manifested by uncontrolled production of antibodies, and / or defects in Cellular self-regulation, leading to a violation of cell apoptosis and the recognition process with loss of immune tolerance to its own antigens, primarily nuclear ones. Among the effector mechanisms of renal damage are complement, polymorphic cells, monocytes, their adhesion factors and attractant molecules, synthesis of cytokines, chemokines, eicosanoids, endothelins, etc., great importance is attached to damage to CD8 - and CD4+ T cells and interstitial macrophages with the subsequent development of fibrosis and loss of peritubular capillaries. To date, a large number of antibodies to various fragments of nuclear structures have been identified. Their role in the pathogenesis of SLE is not equal, but it can determine the clinical features of the disease. Antibodies are directed against nucleic acids and proteins related to intracellular transcription and translation mechanism: their main targets are nucleosomes (DNA histones) or four antigens from their own chromatin, small nuclear ribonucleoproteins (sn RNP) and small cytoplasmic ribonucleoproteins (sc RNP). Antibodies directed against native DNA, Smith (Sm) antigen, and $\mathrm{C}_{1 \mathrm{q}}$ are considered to be diagnostically significant. Antibodies to double-chiral (native) DNA have the greatest specificity and pathogenicity. Antibodies to the igg2b DNA isotype that trigger the classical complement activation pathway are considered potentially nephritogenic. In addition to antibodies to native DNA, importance is attached to other autoantibodies to various cellular structures. Thus, anti-Ro and anti-C1q + antibodies are associated with severe kidney damage. Antibodies that are associated with the development of antiphospholipid syndrome (AFS) - antiphospholipid antibodies (AFA) have a special effect on the clinical picture of the disease and the prognosis of lupus nephritis. AFA is a heterogeneous population of antibodies to antigenic determinants of negatively charged (anionic) phospholipids and / or phospholipid-binding (cofactor) plasma proteins. The AFA family includes: antibodies that cause a false positive Wasserman reaction, antibodies that react 
with cardiolipin $(A C L)$ and other phospholipids, as well as the so - called lupus anticoagulant (VA) - antibodies that lengthen in vitro blood clotting time in phospholipid-dependent coagulation tests. Recently, it has become known that in the implementation of the interaction of AFA with phospholipids, the Central role belongs to mutations of cofactor proteins: $\quad \beta 2$-glycoprotein 1 ( $\left.\beta_{2}-G P \quad 1\right)$, prothrombin, X, XIII blood clotting factor, proteins $\mathrm{C}$ and $\mathrm{S}$, methyltetrahydrofolate reductase, etc. Gene polymorphism of procoagulant proteins and natural anticoagulants can cause the development of macro-and microangiopathies and transform the clinical and morphological picture of nephropathy in SLE $[1,4]$.

The morphology of lupus nephritis is characterized by significant polymorphism both in different glomeruli and within a single glomerulus and is characterized by proliferation of glomerular cells, expansion and interposition of the mesangium, membranous changes, damage to the tubules and interstitium. Specific (although not pathognomonic) morphological features for lupus nephritis are considered to be fibrinoid necrosis of capillary loops, nuclear pathologykaryorexis and karyopycnosis, sharp focal thickening of the basal membranes of the glomerular capillaries in the form of "wire loops". An important element of damage is intravascular thrombosis (fibrin and hyaline clots in the capillary lumen), possibly combined with the presence of AFA or immune complexes containing cryoglobulins. In immunohistochemistry, class G immunoglobulins are detected, mainly IgG1 and $\lg G_{3}$; sometimes, however, IgA or IgM predominates. clinically, lupus nephritis differs From bright's nephritis in its peculiar manifestation and severity of the main nephrological syndromes. With "kidney" masks of the disease, i.e. in cases of SLE debut with kidney damage, such features allow us to suspect the underlying disease, and in the absence of morphological data-to assume the severity of kidney damage and choose an adequate treatment regimen. Features of the main nephrological syndromes in lupus nephritis Proteinuria is an absolute sign of lupus nephritis, is highly non-selective, rarely reaches large values, as in bright nephritis. Nephrotic syndrome - NS) - in lupus nephritis does not have the same prognostic value as in bright nephritis. The 10-year survival rate in patients with NS and severe urinary syndrome is similar, with the exception of cases of onset of the disease with NS. The peculiarity of the latter in lupus nephritis is the rarity of hypovolemia, and the frequent combination of hypertension and hematuria in these patients suggests a frequent combination with acute nephritic syndrome. This explains the lower severity of NS in lupus nephritis and rare hypovolemic crises. Another distinctive feature of NS is a lower tendency to relapse than in bright nephritis. Hematuria is an important criterion for the activity of lupus nephritis, in 2$5 \%$ of cases there is macrohematuria. Pyuria aseptic, with a primary limfozitoza. Renal failure - the rate of increase in serum creatinine is of great importance. Double growth in less than 3 months is a criterion for rapid progression. Acute renal failure is $5-10 \%$. It should be particularly noted that in contrast to bright's nephritis, patients with lupus nephritis in the stage of chronic renal failure often have a high activity of the disease, i.e. lupus nephritis does not always "burn out" even in the presence of clinical signs of uremia and other signs of renal tissue sclerosis, and many patients on program hemodialysis should receive immunosuppressive therapy. Arterial hypertension $(\mathrm{AH})$ occurs in $60-70 \%$ of patients. The frequency of hypertension and the state of 
hemodynamics are closely related to the degree of activity of lupus nephritis. The damaging effect of hypertension on the kidneys, heart, brain, and blood vessels in SLE is compounded by autoimmune damage to these same target organs. Hypertension worsens overall and" renal " survival, increases the risk of death of patients from cardiovascular complications. The reversibility of the increase in blood PRESSURE when remission of lupus nephritis is achieved also confirms the connection of hypertension in this disease with the activity of the process. Nephrosclerosis affects the level of blood PRESSURE only in cases when it reaches significant severity. The risk of developing steroid hypertension in patients with SLE is 8$10 \%$, and with kidney damage-up to $20 \%$. For the development of steroid hypertension, not only the dose, but also the duration of treatment with glucocorticoids (GC) is important. With moderate activity of the process, APS plays a special role as the cause of hypertension.

Depending on the severity of the clinical picture, course and prognosis, the following clinical variants of lupus nephritis are distinguished: active and inactive (table 3) [1].

Table 3. Clinical variants of lupus nephritis

\section{ACTIVE nephritis}

a) fast-progressing;

b) slow-progressing:

c) with nephrotic syndrome;

e) with severe urinary syndrome

2. INACTIVE NEPHRITIS with minimal urinary syndrome or subclinical proteinuria

Vascular lesions can be caused by both the main process and the accompanying AFS. In addition, the early development of atherosclerosis, including with damage to the renal vessels, has been widely discussed recently [9-12]. Clinically and pathomorphologically, vascular lesions are represented by vasculitis and vasculopathy. To vascular lesions of the kidney in Association with APS include the development of nephropathy due to the destruction of large vessels - macroangiopathy (venous and arterial thrombosis, stenosis or occlusion of the renal arteries with the development of renovascular hypertension and/or acute renal failure), and the defeat of the microvasculature within thrombotic microangiopathy intrarenal vessels with ischaemic nephropathy [6-8].

\section{CONCLUSION}

Atherosclerosis and arteriolosclerosis lead to the development of ischemic kidney disease. Treatment tactics depend on the disease activity, clinical and morphological variants of lupus nephritis $[1,13]$. A kidney biopsy is necessary to characterize morphological changes in order to select appropriate therapy, as well as to assess the prognosis of the disease. The activity of therapy should correspond to the activity of the disease: the higher the activity of the process and the more severe the clinical and morphological signs of the disease, the earlier active therapy should be prescribed. Concomitant high hypertension is not a contraindication to the use of intensive treatment, since in most cases it reflects the activity of the process and disappears with remission of the disease. To achieve remission (induction therapy), GC is used in combination with cytostatics. More often, oral administration of GC is combined with pulse therapy, which can increase the effectiveness of the therapy and reduce the risk of complications. The use of cytostatics in lupus nephritis for induction and maintenance therapy is currently recognized as mandatory. 
Among cytostatics in severe cases of the disease, cyclophosphamide (CFA) is preferred according to the intermittent scheme in ultrahigh doses (pulse therapy). After achieving remission, azathioprine (AZA) or selective mycophenolic acid preparations are used instead of CFA for maintenance therapy [14-16]. The duration of maintenance therapy is determined empirically, but even with complete remission of the disease, long-term maintenance therapy is required, often for several years (in most cases, life-long: for women-5 mg, for men-7.5 mg). Despite ongoing clinical trials comparing the effectiveness of General cytostatics (CFA and AZA) and selective action, no convincing data on the benefits of the latter have yet been obtained. However, the results of using supportive treatment regimens for both one and other groups of drugs are comparable. Other selective drugs are also undergoing clinical trials. New strategies for immunological intervention are associated with total irradiation of the lymphoid system or bone marrow, followed by stem cell transplantation. Modern biotechnologies offer a group of anticytokine drugs [antitnfa, anti-interleukin 1receptor: anakinra, anti-interleukin 10, antiinterleukin 6-receptor, anti-interferon $\alpha$, anti Blymphocyte stimulator (Blys)], as well as monoclonal antibodies (anti-CD20: rituximab, anticd22: epratuzumab) and molecular blockers that interrupt specific links of immune recognition. There are the first experimental and clinical experiments with the use of LJP394, which selectively affects the synthesis of antibodies to ds DNA by B-lymphocytes, an inhibitor of the $\mathrm{C}_{5}$-fraction of the complement "Alexion", prostaglandin 1, thromboxane receptor antagonists, $\mathrm{T}$ cell vaccination, etc. The presence of AFS dictates the need to use anticoagulants and antiplatelet agents not only during the period of exacerbation, but also in some cases for life. With the development of end-stage renal failure, hemodialysis or kidney transplantation is performed. In contrast to primary glomerulonephritis, end-stage lupus nephritis may retain high activity of the lupus process, as evidenced by numerous extrarenal symptoms. Therefore, despite the development of nephrosclerosis, in such patients it is necessary to continue immunosuppressive therapy against the background of hemodialysis sessions. Kidney transplantation is performed in the absence of signs of SLE activity $[1,4,5,13]$.

\section{REFERENCES}

1. Tareeva I. E., Krasnova T. N. kidney Damage in systemic lupus erythematosus. Nephrology. A guide for physicians. Ed. by I. E. Tareeva, M.: Medicine, 2000.

2. Vu T. V., Escalante A. a comparison jf quality of life of patients with systemic lupus erythematosus. J Revmatol 1999; 26: 2595-601.

3. Trager J., Ward M.M. Mortality and causes of death in systemic lupus erythematosus. Current Opinion in Rheumatology 2001; 13: 345-51.

4. Cervera R., Khamashta M.A., Font J. Morbidity and mortality in systemic lupus erythematosus during a 10-year period. Medicine 2003; 82(5): 299-308.

5. Cameron J. S. Lupus nephritis and its management in 2001. In: Advances in Nephrology. Edited By N. A. Mukhina, Moscow: Russian doctor, 2001; 145-64.

6. Tareeva I. E., Shilov E. M., Krasnova T. N., etc. Lupus nephritis in the mid-twentieth and early twenty-FIRST century. Ter arch 2001; 6: 5-10.

7. Meteleva N. A., Kozlovskaya N. L. kidney Damage in antiphospholipid syndrome. Ter arch 2004; 9: 91-6. 
8. Mukhin N. A., Kozlovskaya L. V., Kozlovskaya N. L. et al. Primary antiphospholipid syndrome - "venous" and "arterial" variants of the course. In the book: Clinical discussions. Internal disease. Edited By N. A. Mukhin, M.: Litterra, 2005; 261-77.

9. Stenina O. A., Sorokin E. V., Fomicheva O. A., etc. Prevalence and risk factors of atherosclerosis in patients with systemic lupus erythematosus. Cardiology 2005; 11: 105-8.

10. Cao A. N., Sabatine JM, Manzi S. Update on vascular disease in systemic lupus erythematosus. Current Opinion in Rheumatology 2003; 15: 519-27.

11. Kohler H.P., Futers T.S., Grant P.J. Prevalence of three common polymorphisms in the A-subunit gene of factor XIII in patients with coronary artery disease. Thromb Haemost 1999; 81(4): 5115 .

12. Ward M.M. Premature morbidity from cardiovascular and cerebrovascular diseases in women with systemic lupus erythematosus. Arthritis and rheumatism 1999; 42(2): 338-46.

13. Shilov E. M. Lupus nephritis: strategy and treatment. Ter arch 2006; 5: 76-85.

14. D'Crus. Mycophenolate mofetil of systemic vasculitis. Lupus 2005; 14: 55-7.

15. Chan T., Tse K., Tang C. et al. Long-term study of micophenolate mofetil as continuous induction and maintenance treatment for diffuse proliferative lupus nephritis. Am J Soc Dis 2005; 16: 1076-84.

16. Cune W. Mycophenolate mofetil for lupus nephritis. N Engl J Med 2005; 353: 2282-4. 